\title{
Non-Fermi-Liquid-Like Behaviors and Superconductivity Driven by Orbital Fluctuations in Iron Pnictides: Analysis by Fluctuation-Exchange Approximation
}

\author{
Seiichiro ONARI ${ }^{1}$, and Hiroshi KONTANI ${ }^{2}$ \\ ${ }^{1}$ Department of Applied Physics, Nagoya University and JST, TRIP, Furo-cho, Nagoya 464-8602, Japan. \\ ${ }^{2}$ Department of Physics, Nagoya University and JST, TRIP, Furo-cho, Nagoya 464-8602, Japan.
}

(Dated: November 21, 2018)

\begin{abstract}
We study the five-orbital Hubbard-Holstein model for iron pnictides with small electron-phonon interaction due to Fe-ion Einstein oscillators. Using the fluctuation-exchange (FLEX) approximation, orbital fluctuations evolve inversely proportional to the temperature, and therefore the resistivity shows linear or convex $T$-dependence for wide range of temperatures. We also analyze the Eliashberg gap equation, and show that $s$-wave superconducting state without sign reversal $\left(s_{++}\right.$-wave state) emerges when the orbital fluctuations dominate the spin fluctuations. When both fluctuations are comparable, their competition gives rise to a nodal $s$-wave state. The present study offers us a unified explanation for both the normal and superconducting states.
\end{abstract}

PACS numbers: 74.20.-z, 74.20.Fg, 74.20.Rp

The many-body electronic states and the pairing mechanism in iron pnictides have been significant open problems. By taking account of the Coulomb interaction and the nesting of the Fermi surfaces (FSs) in Fig.1 (a), fullygapped sign-reversing $s$-wave state $\left(s_{ \pm}\right.$-wave state) had been proposed $[1,2]$. Experimentally, both $T_{\mathrm{c}}$ and antiferro (AF) spin correlation increases as $x$ decreases in $\mathrm{BaFe}_{2}\left(\mathrm{As}_{1-x} \mathrm{P}_{x}\right)_{2}$ [3]. In contrast, $T_{\mathrm{c}}$ in $\mathrm{LaFeAsO}_{1-x} \mathrm{~F}_{x}$ at $x=0.14$ increases from $23 \mathrm{~K}$ to $43 \mathrm{~K}$ by applying the pressure, whereas AF spin correlation is almost unchanged [4]. Thus, the relationship between $T_{\mathrm{c}}$ and spin fluctuation strength seems to depend on compounds.

On the other hand, orbital-fluctuation-mediated $s$ wave state without sign reversal ( $s_{++}$-wave state) had been proposed based on the five-orbital HubbardHolstein (HH) model $[5,6]$, which is the Hubbard model introduced in Ref. [1] with the addition of the electronphonon (e-ph) interaction term due to Fe-ion Einstein oscillations. Within the random-phase-approximation (RPA), it was found that $d$-orbital fluctuation is induced by small $e$-ph interaction, not by the Coulomb interaction alone. Especially, empirical relation between $T_{\mathrm{c}}$ and the As-Fe-As bond angle (Lee plot) [7] is naturally explained. Recently, theoretically predicated orbital fluctuations $[5,6]$ had been detected via the substantial softening of shear modulus [8]. The $s_{++}$-wave state is consistent with the robustness of $T_{\mathrm{c}}$ against randomness [9-11] as well as the "resonance-like" peak structure in the neutron inelastic scattering [12].

However, spin/orbital fluctuations obtained by the RPA are reduced by the self-energy correction. Therefore, to confirm the orbital fluctuation scenario, it is desired to analyze the many-body electronic states beyond the RPA. For this purpose, the fluctuation-exchange (FLEX) approximation [13] would be appropriate in that the absence of spin/orbital order in 2D systems, known as Mermin-Wagner theorem, is rigorously satisfied [14].

In this letter, we analyze the five-orbital $\mathrm{HH}$ model for iron pnictides using the FLEX approximation. In the normal state, large orbital fluctuations induce highly anisotropic quasiparticle lifetime on the FSs as well as the $T$-linear or $T$-convex resistivity $\rho$ [15-17]. The large orbital fluctuations also introduce the $s_{++}$-wave superconducting (SC) state for wide range of parameters, and the competition between orbital and spin fluctuations lead to the nodal $s$-wave state. We propose that the orbital fluctuation is the origin of both the $s_{++}$-wave SC state and the non-Fermi-liquid-like behavior in the normal state.

In the FLEX approximation [13], the $5 \times 5$ self-energy matrix $\hat{\Sigma}$ in the orbital representation is given by

$$
\Sigma_{l_{1} l_{3}}(k)=\frac{T}{N} \sum_{q} \sum_{l_{2} l_{4}} V_{l_{1} l_{2}, l_{3} l_{4}}^{\Sigma}(q) G_{l_{2} l_{4}}(k-q),
$$

where $N$ is the number of $\boldsymbol{k}$ meshes, and we denote $k=\left(\boldsymbol{k}, \epsilon_{n}\right)$ with fermion Matsubara frequency $\epsilon_{n}=$ $(2 n+1) \pi T$, and $q=\left(\boldsymbol{q}, \omega_{n}\right)$ with $\omega_{n}=2 n \pi T$. Here, $l_{i}$ represents the $Z^{2}, X Z, Y Z, X^{2}-Y^{2}$ and $X Y$ orbitals, which are hereafter denoted as $1,2,3,4$ and 5 , respectively [1]. $\hat{G}$ is the $5 \times 5$ Green function matrix in the orbital basis, and $\hat{V}^{\Sigma}$ is the $25 \times 25$ interaction term for the self-energy given as [18]

$$
\begin{aligned}
\hat{V}^{\Sigma}(q) & =\frac{3}{2} \hat{\Gamma}^{s} \hat{\chi}^{s}(q) \hat{\Gamma}^{s}+\frac{1}{2} \hat{\Gamma}^{c} \hat{\chi}^{c}(q) \hat{\Gamma}^{c} \\
& -\frac{1}{4}\left(\hat{\Gamma}^{s}-\hat{\Gamma}^{c}\right) \hat{\chi}^{\operatorname{irr}}(q)\left(\hat{\Gamma}^{s}-\hat{\Gamma}^{c}\right)+\frac{3}{2} \hat{\Gamma}^{s}+\frac{1}{2} \hat{\Gamma}^{c}
\end{aligned}
$$

where the irreducible susceptibility is given by

$$
\chi_{l_{1} l_{2}, l_{3} l_{4}}^{\mathrm{irr}}(q)=-\frac{T}{N} \sum_{k} G_{l_{1} l_{3}}(k+q) G_{l_{4} l_{2}}(k)
$$

and the spin (orbital) susceptibility is obtained as $\hat{\chi}^{s(c)}=$ $\hat{\chi}^{\text {irr }}\left(1-\hat{\Gamma}^{s(c)} \hat{\chi}^{\text {irr }}\right)^{-1}$. Here, $\hat{\Gamma}^{s}=\hat{S}\left(\hat{\Gamma}^{c}=-\hat{C}-2 \hat{V}\left(\omega_{n}\right)\right)$ is the irreducible vertex for spin (charge) channel; $\hat{S}$ and $\hat{C}$ represent the Coulomb interaction in the multiorbital 
model introduced in Refs. [1, 5, 6, 18]; Their matrix elements consist of the intra-orbital Coulomb $U$, the interorbital Coulomb $U^{\prime}$, Hund's coupling $J$ and the pair hopping $J^{\prime}$. Here we assume that $J=J^{\prime}$ and $U=U^{\prime}+2 J$.

The vertex $\hat{V}\left(\omega_{n}\right)$ in $\hat{\Gamma}^{c}$ represents the electron-electron (el-el) interaction mediated by $e$-ph interaction. For example, we show non-zero $V_{l l^{\prime}, m m^{\prime}}$ for $l, l^{\prime}, m, m^{\prime}=2,3,4$ in Fig. 1 (b), where $g\left(\omega_{n}\right)=g \omega_{\mathrm{D}}^{2} /\left(\omega_{n}^{2}+\omega_{\mathrm{D}}^{2}\right)$ is proportional to the phonon Green function; $g=g(0)$ is the effective el-el interaction for $\omega_{n}=0$, and $\omega_{\mathrm{D}}$ is the Debye frequency [5]. Other than Fig. 1 (b), $\hat{V}$ has many non-zero off-diagonal elements as explained in Ref. [6], since the Fe-ion oscillation (non- $\mathrm{A}_{1 g}$ mode) induces various inter-orbital transitions. This fact gives rise to the prominent orbital fluctuations at low frequencies, while the charge susceptibility $\chi^{c}(\boldsymbol{q})=\sum_{l m} \chi_{l l, m m}^{c}(\boldsymbol{q})$ is not enhanced due to the cancellation $[5,6]$. In the present study, we drop ladder-type diagrams by $g\left(\omega_{n}\right)$, which is justified when $\omega_{\mathrm{D}} \ll E_{\mathrm{F}}[5,6]$. For the same reason, $g\left(\omega_{n}\right)$ is absent in $\hat{\Gamma}^{s}$.

In the FLEX approximation, we obtain $\hat{G}$ and $\hat{\Sigma}$ selfconsistently using the Dyson equation $\hat{G}^{-1}=\left(\hat{G}^{0}\right)^{-1}-\hat{\Sigma}$. In multiband systems, the FSs are modified from the original FSs due to the self-energy correction. To escape from this difficulty, we subtract the constant term $[\hat{\Sigma}(\boldsymbol{k},+i 0)+$ $\hat{\Sigma}(\boldsymbol{k},-i 0)] / 2$ from the original self-energy, corresponding to the elimination of double-counting terms between LDA and FLEX [19]. Hereafter, we fix $J / U=1 / 6$, $\omega_{\mathrm{D}}=0.02 \mathrm{eV}$, and the electron filling $n=6.1$ except for Fig. 4. Because of the smallness of the FSs in Fig. 1, fine $\boldsymbol{k}$ meshes are required for a quantitative study. We take $N=128 \times 128 \boldsymbol{k}$ meshes that is four times of that used in Ref. [19], and 1024 Matsubara frequencies. Then, we obtain reliable numerical results for $T \geq 0.01 \mathrm{eV}$. (a) Fermi surface

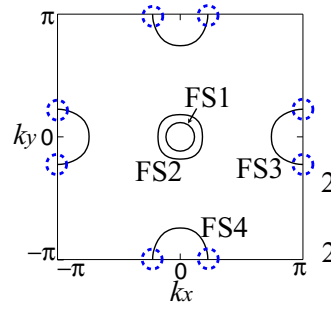

(b) el-el interaction $\hat{V}\left(\omega_{n}\right)$
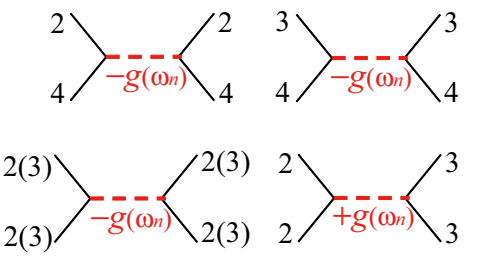

FIG. 1: (color online) (a) FSs in the unfolded zone. The dotted circles represent the cold-spot given by the orbital fluctuation theory. The cold-spot is composed of $x z / y z$-orbitals. (b) Phonon-mediated el-el interaction $(\hat{V})$ for $2,3,4$ orbitals.

We begin with the electronic property in the normal state. Hereafter, the unit of energy is eV. First, we discuss the quasiparticle damping rate $\gamma_{\boldsymbol{k}}$ on each FS, which is given by the imaginary part of the self-energy in the band-diagonal representation. In Fig. $2(\mathrm{a}), \gamma_{\boldsymbol{k}}^{s(c)}$ represents the damping due to spin (orbital) fluctuations for $T=0.015$ and $U=1.8$, which is given by substituting
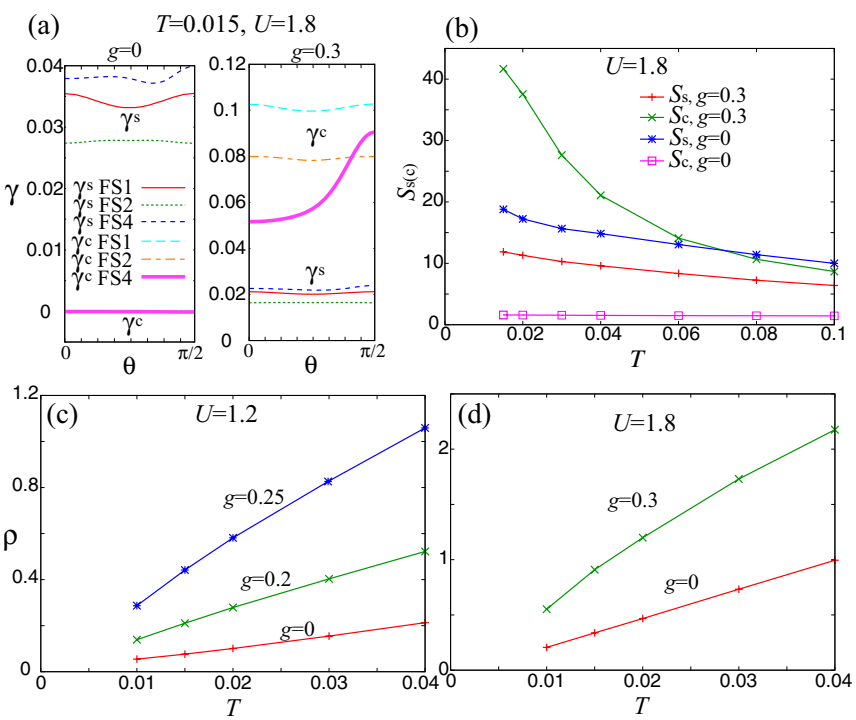

FIG. 2: (color online) (a) $\boldsymbol{k}$-dependence of $\gamma^{s(c)}$ induced by the spin (orbital) fluctuations on each FS. (b) $T$-dependence of $S_{s(c)}=\left(1-\alpha_{s(c)}\right)^{-1}$. (c),(d) $T$-dependence of $\rho$. $\rho=$ 1 corresponds to $\left(\hbar a_{c}\right) / e^{2} \sim 300 \mu \mathrm{cm} \Omega$ when the interlayer distance is $a_{c}=0.6 \mathrm{~nm}$.

$\hat{V}^{\Sigma}=\frac{3}{2} \hat{\Gamma}^{s} \hat{\chi}^{s} \hat{\Gamma}^{s}\left(\frac{1}{2} \hat{\Gamma}^{c} \hat{\chi}^{c} \hat{\Gamma}^{c}\right)$ in eq. (1). The horizonal axis is the azimuth angle for $\boldsymbol{k}$ point with the origin at $\Gamma(\mathrm{M})$ point for FS1,2 (FS4). The relation $\gamma_{\boldsymbol{k}} \approx \gamma_{\boldsymbol{k}}^{s}+\gamma_{\boldsymbol{k}}^{c}$ is satisfied since the third term in eq. (2) is very small. We will see below that the value $U=1.8$ can reproduce moderate $\mathrm{AF}$ spin fluctuations observed in $e$-doped compounds, and it is consistent with $U \sim 2$ reported by x-ray absorption spectroscopy (XAS) [20].

In the case of $g(0)=g=0$, the relation $\gamma^{s} \gg \gamma^{c}$ holds since the orbital fluctuation is very small. However, $\gamma^{c}$ gradually develops with $g$, and $\gamma^{s} \sim \gamma^{c}$ at $g=0.26$. In Fig. $2(\mathrm{a}), \gamma^{c} \gg \gamma^{s}$ at $g=0.3$; the corresponding dimensionless coupling is just $\lambda \equiv g N(0) \sim 0.2$. In contrast, $\gamma^{s}$ decreases with $g$, due to the suppression of $\hat{\chi}^{s}$ by $\gamma^{c}$.

As shown in Fig. 2 (a), the momentum dependence of $\gamma_{\boldsymbol{k}}^{s}$ on each FS is small, although AF spin correlation is well developed. In contrast, $\gamma_{\boldsymbol{k}}^{c}$ on FS4 (e-pockets) takes the minimum value at $\theta \sim 0$ for $g=0.3$; this "coldspot" is important for the transport phenomena. Since the cold spot is on the e-pocket, the Hall coefficient $R_{\mathrm{H}}$ will be negative, which is consistent with experiments $[15,16,21]$. In the case of high- $T_{\mathrm{c}}$ cuprates, various nonFermi-liquid-like transport phenomena (e.g., violation of Kohler's rule) originate from the cold/hot spot structure as well as the backflow (=current vertex correction) due to spin fluctuations [22]. Therefore, appearance of the cold spot in Fig. 2 (a) indicates that the orbital fluctuations are the origin of striking non-Fermi-liquid-like transport phenomena in iron pnictides [15, 16, 21].

In Fig. 2 (b), we show how the orbital and spin fluctuations develop as $T$ decreases: In the FLEX, the 
spin (orbital) susceptibility is enhanced by the spin (orbital) Stoner enhancement factor $S_{s(c)}=\left(1-\alpha_{s(c)}\right)^{-1}$, where $\alpha_{s(c)}$ is the maximum of the largest eigenvalue of $\hat{\Gamma}^{s(c)} \hat{\chi}^{\operatorname{irr}}(\boldsymbol{q}, 0)$ with respect to $\boldsymbol{q}$. Then, $\alpha_{s, c}=1$ corresponds to the spin/orbital order, although it is prohibited in 2D systems by the Mermin-Wagner theorem [14]. In case of $U=1.8$ and $g=0$, large $S_{s}(\gtrsim 10)$ is induced at $\boldsymbol{q} \approx \boldsymbol{Q} \equiv(\pi, 0)$ (i.e., $\left.\chi^{s}(\boldsymbol{Q}, 0) \propto S_{s}\right)$. $S_{s}$ gradually increases as $T$ drops, which is a typical critical behavior near the AF magnetic quantum-critical-point (QCP) [23]. When $g>0, \chi^{c}(\boldsymbol{q}, 0)$ is enhanced at $\boldsymbol{q}=\mathbf{0}$ and $\boldsymbol{q}=\boldsymbol{Q}$ almost equivalently [6]. At $g=0.3$, large $S_{c}(\gg 10)$ is induced at both $\boldsymbol{q} \approx \boldsymbol{Q}$ and $\mathbf{0}$, and it increases approximately proportional to $T^{-1}$. Thus, it is confirmed that both ferro- and AF-orbital fluctuations show critical evolusions near the orbital QCP.

Next, we discuss the resistivity $\rho$ due to the orbital and spin fluctuations. By neglecting the backflow, the conductivity is obtained by

$\sigma_{x x}=\frac{1}{N} \sum_{\boldsymbol{k}, \alpha} \int_{-\infty}^{\infty} \frac{d \omega}{\pi}\left(-\frac{\partial f(\omega)}{\partial \omega}\right)\left|v_{\alpha, \boldsymbol{k}}^{x} G_{\boldsymbol{k}, \alpha}(\omega+i 0)\right|^{2}$

where $\alpha$ is the band index, $f(\omega)$ is the Fermi distribution function, $v_{\alpha, \boldsymbol{k}}^{x}$ is the velocity of band $\alpha$, and $G_{\boldsymbol{k}, \alpha}(\omega+i 0)$ is the retarded Green function for band $\alpha$ in the FLEX approximation. Figure 2 (c) and (d) show the obtained the resistivity $\rho=1 / \sigma_{x x}$ for $U=1.2$ and 1.8: In case of $U=1.2, \rho$ shows a conventional sublinear (concave) $T$ dependence at $g=0 . \rho$ increases with $g$ due to the orbital fluctuations, and almost $T$-linear resistivity is realized at $g=0.2$. At $g=0.25, \rho$ shows a superlinear (convex) $T$-dependence. In case of $U=1.8, \rho$ is linear-in- $T$ at $g=0$, while it shows a clear superlinear $T$-dependence at $g=0.3$. Thus, we stress that non-Fermi-liquid resistivity is not a direct evidence for the spin fluctuations. In $L n \mathrm{FeAsO}$ compounds, $T_{\mathrm{c}}$ increases as the radius of ranthanide ion $\mathrm{Ln}^{3+}$ decreases, and the $T$-dependence of $\rho$ changes from concave to convex [17]. This experimental correlation between $T_{\mathrm{c}}$ and $\rho(T)$ may be understood in terms of the orbital fluctuation scenario.

Next, we discuss the SC state. In the presence of dilute impurities $\left(n_{\mathrm{imp}} \ll 1\right)$, the linearized Eliashberg equation in the orbital basis is [5]:

$$
\begin{aligned}
\lambda_{\mathrm{E}} \Delta_{l l^{\prime}}(k)= & -\frac{T}{N} \sum_{k^{\prime}, m_{i}} W_{l m_{1}, m_{4} l^{\prime}}\left(k-k^{\prime}\right) G_{m_{1} m_{2}}^{\prime}\left(k^{\prime}\right) \\
& \times \Delta_{m_{2} m_{3}}\left(k^{\prime}\right) G_{m_{4} m_{3}}^{\prime}\left(-k^{\prime}\right)+\delta \Sigma_{l l^{\prime}}^{a}\left(\epsilon_{n}\right),(5)
\end{aligned}
$$

where $\Delta_{l l^{\prime}}(k)$ is the gap function and $\lambda_{\mathrm{E}}$ is the eigenvalue that reaches unity at $T=T_{c} . \delta \hat{S}$ igma ${ }^{a}$ represents the impurity-induced gap function. $\left(\hat{G}^{\prime}\right)^{-1}=(\hat{G})^{-1}-\delta \hat{\Sigma}^{n}$, where $G$ is the Green function given by eq. (1), and $\delta \hat{\Sigma}^{n}$ is the impurity-induced normal self-energy. The pairing interaction $\hat{W}$ in eq. (5) is

$$
\hat{W}(q)=\frac{3}{2} \hat{\Gamma}^{s} \hat{\chi}^{s}(q) \hat{\Gamma}^{s}-\frac{1}{2} \hat{\Gamma}^{c} \hat{\chi}^{c}(q) \hat{\Gamma}^{c}+\frac{1}{2} \hat{\Gamma}^{s}-\frac{1}{2} \hat{\Gamma}^{c},
$$

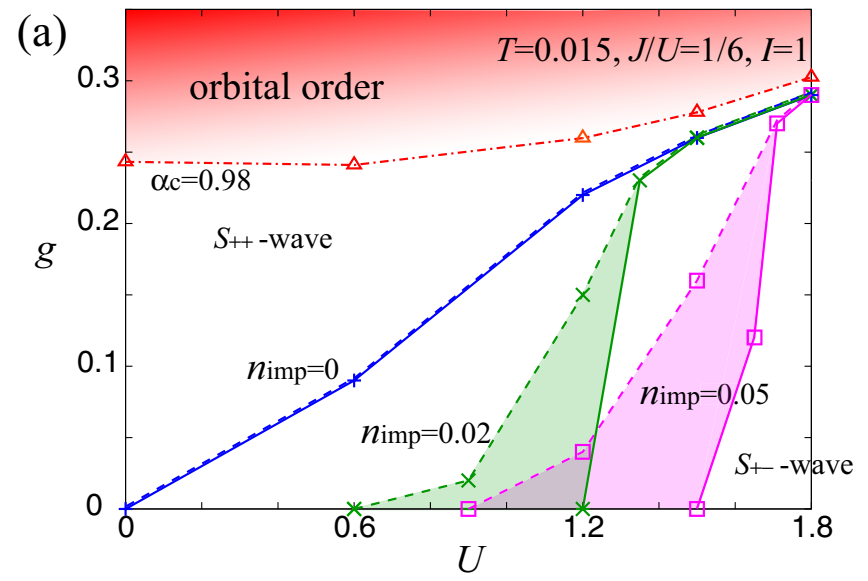

(b) $n_{\text {imp }}=0$

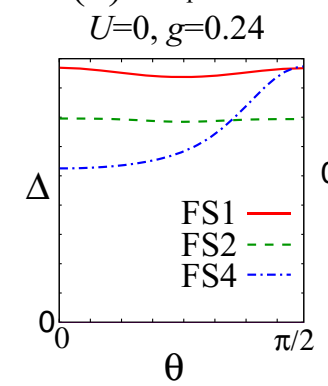

(c) $n$ imp $=0$

(d) $n$ imp $=0.02$

$U=1.8, g=0$

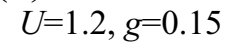

FIG. 3: (color online) (a) $U-g$ phase diagram given by solving the linearized Eliashberg equation at $T=0.015$. Nodal $s$ wave gap state is obtained in the shaded area for $n_{\mathrm{imp}}=0.02$ and 0.05 , and solid lines (dotted lines) represent the boundary between fully-gapped $s_{+-- \text {wave }}\left(s_{++}\right.$-wave) state. Dasheddotted line denotes $\alpha_{c}=0.98$. (b) $s_{++}$-wave gap $\left(\lambda_{E}=0.59\right)$ for $U=0$ and $g=0.24$. (c) $s_{+-}$-wave gap $\left(\lambda_{E}=0.49\right)$ for $U=1.8$ and $g=0$ and. (d) Nodal $s$-wave gap $\left(\lambda_{E}=0.28\right)$ for $U=1.2$ and $g=0.15$.

where $\hat{\chi}^{s, c}$ is given by the FLEX approximation for $n_{\text {imp }}=0$, because of the fact that the fully self-consistent FLEX with impurity-induced self-energy leads to unphysical reduction in $\chi^{s}$, unless vertex correction is taken into account [22]. The first (second) term in eq. (6) works to set $\Delta_{\mathrm{FS} 1,2} \cdot \Delta_{\mathrm{FS} 3,4}<0(>0)$.

In the $T$-matrix approximation, $\delta \hat{\Sigma}^{n, a}$ is given as

$$
\begin{aligned}
& \delta \Sigma_{i j}^{n}\left(\epsilon_{n}\right)=n_{\mathrm{imp}} T_{i j}\left(\epsilon_{n}\right), \\
& \delta \Sigma_{i j}^{a}\left(\epsilon_{n}\right)=n_{\mathrm{imp}} \sum_{l m} T_{i l}\left(\epsilon_{n}\right) f_{l m}\left(\epsilon_{n}\right) T_{j m}\left(-\epsilon_{n}\right),
\end{aligned}
$$

where $T_{i j}\left(\epsilon_{n}\right) \equiv I\left(1-I \hat{g}\left(\epsilon_{n}\right)\right)^{-1}$ is the $T$-matrix in the normal state $[9] ; \hat{g}\left(\epsilon_{n}\right) \equiv \frac{1}{N} \sum_{\boldsymbol{k}} \hat{G}_{\boldsymbol{k}}\left(\epsilon_{n}\right)$ is the local normal Green function, and $I$ is the local impurity potential that is diagonal in the orbital basis. We put $I=1$ hereafter. In eq. (8), $f_{i j}\left(\epsilon_{n}\right)=$ $\frac{1}{N} \sum_{\boldsymbol{k}, l m} G_{i l}(k) \Delta_{l m}(k) G_{j m}(-k)$ is the linearized local anomalous Green function.

In Fig. 3 (a), we show the $U-g$ phase diagram obtained by the FLEX approximation. The dashed-dotted 
line represents the condition $\alpha_{c}=0.98$ at $T=0.015$, corresponding to $g=0.25 \sim 0.3$. (In the RPA, the same condition is satisfied for $g=0.21 \sim 0.23[6]$.) Therefore, prominent orbital fluctuations emerge for $\lambda=g N(0) \lesssim$ 0.2 even if the self-energy correction is taken into account. On the other hand, $\alpha_{s}=0.95$ (0.92) for $U=1.8$ and $g=0(0.3)$ in the FLEX approximation, although $U_{\text {cr }}=1.25$ for $\alpha_{s}=1$ in the RPA. Thus, the renormalization in $\alpha_{s}$ is rather larger than that in $\alpha_{c}$, because of the difference in the coefficients (in factor 3) between the first and the second terms in eq. (2).

Next, we solve eq. (5) with high accuracy using the Lanczos method at $T=0.015$. Then, $s_{++}$-wave gap function is obtained around the line $\alpha_{c}=0.98$; Figure 3 (b) shows the $s_{++}$-wave gap for $g=0.24$ and $U=0$ $\left(\lambda_{E}=0.59\right)$. On the other hand, $s_{ \pm}$-wave gap is obtained when $g$ is sufficiently small; Figure 3 (c) shows the $s_{ \pm}$-wave gap for $U=1.8$ and $g=0\left(\lambda_{E}=0.49\right)$. When $n_{\text {imp }}=0$, the gap function changes from (b) to (c) discontinuously on the phase boundary in Fig. 3 (a), as found in Ref. [6]. When $n_{\mathrm{imp}} \geq 0.02$, however, gap function changes smoothly during the crossover. Then, line-nodes inevitably appear on FS3,4 in the shaded area in Fig. 3 (a); Figure 3 (d) shows the nodal $s$-wave gap for $U=1.2, g=0.15$ and $n_{\mathrm{imp}}=0.02\left(\lambda_{E}=0.28\right)$. Thus, both regions for $s_{++}$-wave and nodal $s$-wave states are extended by small amount of impurities, although $\lambda_{E}$ for the latter state is reduced by impurities. A nodal $s$-wave solution at $n_{\text {imp }}=0$ with larger $\lambda_{E}$ may be obtained by considering a three-dimensional (3D) nodal-line structure in 3D tight-binding model [24].

Here, we discuss that line nodes originate from the competition between the orbital and spin fluctuations: The electrons at $\theta \sim 0(\pi / 2)$ on FS4 is composed of orbital 2,3 (4). Since the orbital 4 is absent in FS1,2, the nesting-driven spin correlation between the orbital 2,3 on FS1,2 and the orbital 4 on FS3,4 is weak. (That is, $\chi_{24,42}^{s}(\boldsymbol{Q}) \ll \chi_{22,22}^{s}(\boldsymbol{Q})$.) On the other hand, both $\chi_{24,42}^{c}(\boldsymbol{q})$ and $\chi_{22,22}^{c}(\boldsymbol{q})$ are developed here [5, 6]. Therefore, when orbital and spin fluctuations are comparable, $\Delta_{\mathrm{FS} 1,2} \cdot \Delta_{\mathrm{FS} 4}$ is negative (positive) at $\theta \sim 0(\pi / 2)$ due to the orbital-dependence on the spin correlation.

In Fig. 4 (a), we show the filling dependence of $\lambda_{\mathrm{E}}$ for the $s_{++}$-wave state $(U=1.2, g=0.25)$, and that for the $s_{+-}$-wave state $(U=1.8, g=0)$. We note that FS1,2 disappear for $n>6.3$. The value of $\lambda_{\mathrm{E}}$ for the $s_{++}$-wave state decreases monotonically with $n$, while $\lambda_{\mathrm{E}}$ for the $s_{+-}$-wave state is rather insensitive to $n$, maybe because the temperature, $T=0.02$, is rather high. Figure 4 (b) shows that $\lambda_{\mathrm{E}}$ for the $s_{++}$-wave state $(U=1.2, n=6.1)$ increases with $g$.

In summary, we performed the FLEX approximation in the multiorbital $\mathrm{HH}$ model for iron pnictides. It was confirmed that orbital-fluctuation-mediated $s_{++}$-wave state is realized by small $e$-ph interaction $g$. As increasing the value of $g$, both the $T_{\mathrm{c}}$ of $s_{++}$-wave state and the

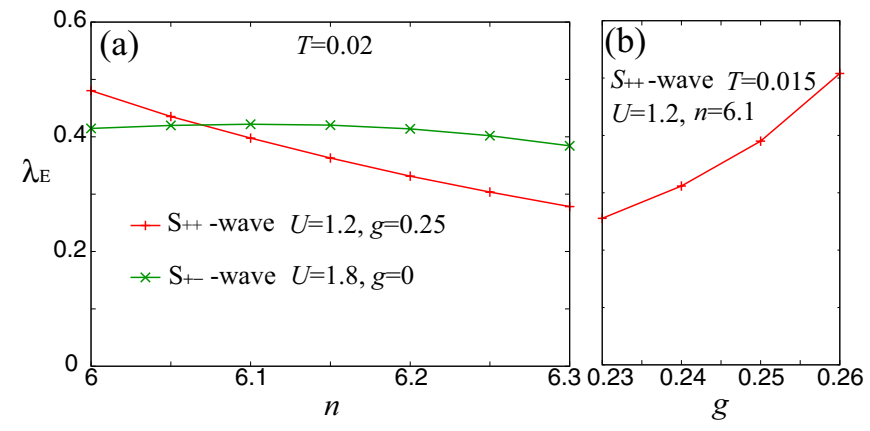

FIG. 4: (color online) (a) $n$ dependence of $\lambda_{\mathrm{E}}$ for $s_{++^{-}}$and

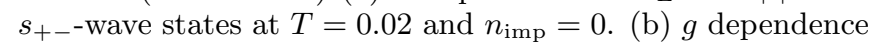
of $\lambda_{\mathrm{E}}$ for $s_{++}$-wave state at $T=0.015$ and $n_{\mathrm{imp}}=0$.

resistivity $\rho$ are increased, and the latter changes from $T$-concave to $T$-convex. This correlation between $T_{\mathrm{c}}$ and $\rho$ is consistent with experiment [17]. The region of $s_{++^{-}}$ wave or nodal $s$-wave states is enlarged in the presence of small amount of impurities. Thus, the present orbital fluctuation scenario presents a unified explanation for both normal and SC electronic states.

We are grateful to M. Sato, Y. Kobayashi, Y. Matsuda, T. Shibauchi, D.S. Hirashima, Y. Tanaka, K. Yamada, and F.C. Zhang for valuable discussions. This study has been supported by Grants-in-Aid for Scientific Research from MEXT of Japan, and by JST, TRIP.

[1] K. Kuroki et al., Phys. Rev. Lett. 101, 087004 (2008).

[2] I.I. Mazin et al.: Phys. Rev. Lett. 101, 057003 (2008).

[3] Y. Nakai et al., Phys. Rev. Lett. 105, 107003 (2010).

[4] T. Nakano et al., Phys. Rev. B 81, 100510(R) (2010).

[5] H. Kontani and S. Onari, Phys. Rev. Lett. 104, 157001 (2010).

[6] T. Saito, S. Onari and H. Kontani, arXiv:1008.1765.

[7] C.-H. Lee et al., J. Phys. Soc. Jpn. 77, 083704 (2008); Y. Mizuguchi et al., Supercond. Sci. Technol. 23, 054013 (2010).

[8] M. Yoshizawa et al., arXiv:1010.1479.

[9] S. Onari, and H. Kontani, Phys. Rev. Lett. 103, (2009) 177001.

[10] A. Kawabata et al., J. Phys. Soc. Jpn. 77, 103704 (2008).

[11] Y. Nakajima et al., arXiv:1009.2848.

[12] S. Onari et al., Phys. Rev. B 81, 060504(R) (2010).

[13] N. E. Bichers, D. J. Scalapino, Ann. Phys. (N.Y.) 193, 206 (1989).

[14] H. Kontani and M. Ohno, Phys. Rev. B 74, 014406 (2006).

[15] R. H. Liu et al., Phys. Rev. Lett. 101, 087001 (2008).

[16] Kasahara et al., Phys. Rev. B 81, 184519 (2010).

[17] K. Miyazawa et al., J. Phys. Soc. Jpn. 78, 034712 (2010); S. Ishida et al., Phys. Rev. B 81, 094515 (2010).

[18] T. Takimoto et al., Phys. Rev. B 69, 104504 (2004).

[19] H. Ikeda et al, Phys. Rev. B 81, 054502 (2010).

[20] W. L. Yang et al., Phys. Rev. B 80, 014508 (2009).

[21] S.C. Lee et al, J. Phys. Soc. Jpn. 78, 043703 (2009). 
[22] H. Kontani, Rep. Prog. Phys. 71 (2008) 026501.

[24] I.I. Mazin et al., arXiv:1008.0032.

[23] T. Moriya and K. Ueda, Adv. Phys. 49, 555 (2000). 